\title{
Impacto de las políticas de salud en la incidencia de reperfusión de infarto agudo de miocardio durante el inicio de la pandemia por COVID-19 en Uruguay
}

\author{
Dres. Víctor Dayan ${ }^{1,2}$, Abayubá Perna ${ }^{3}$, Enrique Soto $^{1+}$, Álvaro Niggemeyer ${ }^{1,2}$, \\ Alejandro Cuesta ${ }^{1,2}$, Natalia Piñeiro ${ }^{3}$, Graciela Fernández ${ }^{3}$, Rosana Gambogi ${ }^{3}$
}

\section{Resumen}

Introducción: las medidas sanitarias de emergencia impuestas para contener el SARS-CoV-2 pueden tener efectos colaterales en la atención de enfermedades cardiovasculares. Los datos mundiales de los países sobre la incidencia de infarto agudo de miocardio con elevación del segmento ST (IAMCEST) durante la pandemia son fundamentales para la política sanitaria futura.

Objetivos: nuestro objetivo fue determinar si las medidas sanitarias de emergencia impuestas en Uruguay tuvieron un impacto directo en la calidad de la atención en la reperfusión del IAMCEST.

Métodos: realizamos un estudio retrospectivo poblacional de todo el país para determinar la incidencia de reperfusión de IAMCEST (fibrinolíticos e intervención coronaria percutánea, FBL e ICP respectivamente) durante el período sanitario de emergencia. La tasa de incidencia de la reperfusión, el tiempo hasta la reperfusión y la mortalidad asociada se recopilaron de la base de datos del Fondo Nacional de Recursos (organización gubernamental única a cargo de la financiación de la reperfusión del IAMCEST en Uruguay). Estos mismos datos se recuperaron para 2019, 2018 y 2017.

Resultados: se trataron durante el periodo de estudio del 2020 (136 pacientes) en comparación con 2019 (180 pacientes), 2018 (182 pacientes) y 2017 (174 pacientes). Se realizó FBL como tratamiento único en 5,1\%, 7,2\%, 7,7\% y $12,1 \%$, respectivamente. La razón de tasa de incidencia de IAMCEST durante el período estudiado en 2020 fue de 0,7 (IC95\%: 0,59-0,91). La mediana del tiempo hasta la reperfusión fue similar en comparación con 2019, 2018 y 2017 $(p=0,4)$. No hubieron diferencias en la mortalidad a 15 dias entre los años evaluados.

Conclusión: las medidas sanitarias de emergencia se asociaron con una disminución en la incidencia de reperfusión de IAMCEST, sin afectar el tiempo de reperfusión ni la mortalidad.

Palabras clave: COVID-19

\section{IAMCEST}

EPIDEMIOLOGÍA

\section{Impact of public health policies on the incidence of acute myocardial reperfusion during the initial stages of the COVID-19 pandemic in Uruguay}

\section{Summary}

Introduction: the emergency health measures imposed to contain SARS-CoV-2 can have collateral effects in the care of cardiovascular diseases. Global country data on the incidence of ST acute myocardial infarction during the pandemic are critical for future health policy.

Objectives: our objective was to determine if the emergency health measures imposed in Uruguay had a direct impact on the quality of ST elevation acute myocardial infarction care.

Methods: we carried out a population-based retrospective study of the entire country to determine the incidence of

1. Sociedad Uruguaya de Cardiología. Montevideo, Uruguay.

2. Centro Cardiovascular Universitario. Universidad de la República. Montevideo, Uruguay.

3. Fondo Nacional de Recursos. Montevideo, Uruguay.

Correspondencia: Víctor Dayan. 26 de Marzo 3459/602. Correo electrónico: victor_dayan@hotmail.com.

Ninguno de los autores presenta conflictos de interés.

Recibido Abr 23, 2021; aceptado Oct 4, 2001 
reperfusion of ST elevation acute myocardial infarction (fibrinolytic and percutaneous) during the emergency health period. The incidence rate of reperfusion, time to reperfusion, and associated mortality were collected from the Fondo Nacional de Recursos (the only government organization in charge of the reperfusion of ST elevation myocardial infarction in Uruguay). These same data were recovered for 2019, 2018 and 2017.

Results: fewer patients were treated in 2020 (136 patients) compared to 2019 (180 patients), 2018 (182 patients), and 2017 (174 patients). Fibrinolytics was performed as the only treatment in $5.1 \%, 7.2 \%, 7.7 \%$ and $12.1 \%$ respectively. The proportion in incidence rate of ST elevation myocardial infarction during the study period in 2020 was lower (0.74, 95\% CI: 0.59-0.91). The median time to reperfusion was similar compared to 2019, 2018, and 2017 ( $\mathrm{p}=$ 0.4). Mortality at 15 days was similar in 2017 (8\%), 2018 (6\%), 2019 (11\%) and 2020 (8\%).

Conclusion: emergency health measures were associated with a decrease in the incidence of reperfusion of ST elevation myocardial infarction without affecting the time to reperfusion and mortality.

Key words: $\quad$ COVID-19

STEMI

EPIDEMIOLOGY

\section{Impacto das políticas de saúde na incidência de infarto do miocárdio durante a pandemia de COVID-19 no Uruguai}

\section{Resumo}

Introdução: as medidas emergenciais de saúde impostas para conter o SARS-CoV-2 podem ter efeitos colaterais no cuidado das doenças cardiovasculares. Os dados globais do país sobre a incidência de infarto agudo do miocárdio durante a pandemia são essenciais para a futura política de saúde.

Objetivos: nosso objetivo foi determinar se as medidas de saúde de emergência impostas no Uruguai tiveram um impacto direto na qualidade do atendimento infarto agudo do miocárdio.

Métodos: foi realizado um estudo retrospectivo de base populacional em todo o país para determinar a incidência de reperfusão do infarto agudo do miocárdio (fibrinolítico e percutâneo) durante o período de emergência de saúde. A taxa de incidência de reperfusão, tempo de reperfusão e mortalidade associada foram coletados do Fondo Nacional de Recursos (a única organização governamental responsável pela reperfusão de infarto agudo do miocárdio no Uruguai). Esses mesmos dados foram recuperados para 2019, 2018 e 2017.

Resultados: menos pacientes foram tratados em 2020 (136 pacientes) em comparação com 2019 (180 pacientes), 2018 (182 pacientes) e 2017 (174 pacientes). Fibrinolisis foi realizado como o único tratamento em 5,1\%, 7,2\%, 7,7\% e $12,1 \%$, respectivamente. A proporção na taxa de incidência de infarto agudo do miocárdio durante o período estudado em 2020 foi menor (0,74, IC 95\%: 0,59-0,91). O tempo médio para reperfusão foi semelhante em comparação com 2019, 2018 e 2017 ( $\mathrm{p}$ = 0,4). A mortalidade em 15 dias foi semelhante em 2017 (8\%), 2018 (6\%), 2019 (11\%) e 2020 (8\%). Conclusão: as medidas emergenciais de saúde foram associadas à diminuição da incidência de reperfusão do infarto agudo do miocárdio, sem afetar o tempo de reperfusão e a mortalidade.

Palavras chave: COVID-19

IAMCEST

EPIDEMIOLOGIA

\section{¿Qué aporta este estudio al conocimiento actual?}

La pandemia por SARS-CoV-2 ha determinado una disminución en la incidencia de SCA. Varios centros han reportado aumento de la mortalidad asociada a los IAMCEST durante dicho período.

Datos globales de Uruguay muestran una disminución significativa en el tratamiento de reperfusión de IAMCEST durante el inicio de la pandemia. Esta disminución no se asoció a mayor mortalidad ni mayor tiempo a la reperfusión, lo que contrasta con gran parte de las publicaciones del tema.

\section{Introducción}

Las medidas sanitarias de emergencia se implementaron en Uruguay el 13 de marzo del 2020 luego de que se reportara el primer grupo de cuatro casos de SARS-CoV-2. Desde entonces, se han impulsado medidas de salud pública a gran escala como el distanciamiento físico, el cierre de oficinas públicas, entidades de enseñanza y grandes centros comerciales. Aunque estas medidas han ayudado a limitar la difusión de la infección por SARS-CoV-2 a 984 casos hasta el 29 de mayo del 2020, probablemente hayan afectado la expresión y/o el tratamiento de otras enfermedades como los síndromes coronarios agudos (SCA) ${ }^{(1-6)}$. 
En Uruguay, el tratamiento de intervención coronaria percutánea (ICP) y fibrinolítica (FBL) para el infarto agudo de miocardio con elevación del segmento ST (IAMCEST) está cubierto por una única entidad pública no estatal llamada Fondo Nacional de Recursos (FNR).

Considerando la baja incidencia de SARSCoV-2 en nuestro país, describimos el impacto de las medidas sanitarias de emergencia en la atención del IAMCEST en las etapas iniciales de la pandemia. Nos centramos en las siguientes variables: 1) tasa de incidencia de la terapia de reperfusión con IAMCEST; 2) tiempo desde el inicio de los síntomas hasta la reperfusión; y 3) mortalidad a 15 días para los pacientes que se sometieron a tratamiento por IAMCEST.

\section{Métodos}

Realizamos un estudio descriptivo y analítico retrospectivo poblacional con el objetivo de evaluar la tasa de terapia de reperfusión de IAMCEST desde el 13 de marzo de 2020 hasta el 30 de abril de 2020 en Uruguay. Se recopilaron los mismos datos para 2019, 2018 y 2017. Incluimos a todos los pacientes en Uruguay que recibieron FBL o ICP. Los datos se extrajeron de la base de datos del FNR, que no incluye a pacientes que pudieran haber sufrido un IAMCEST y no hayan recibido reperfusión.

Se definió IAMCEST como la presencia de dolor precordial y elevación de $2 \mathrm{~mm}$ del segmento ST en dos o más derivadas contiguas.

Se comparó la edad, presencia de hipertensión, diabetes y la procedencia (Montevideo vs. fuera de Montevideo).

Se calcularon las tasas de mortalidad con su IC de $95 \%$ y se compararon mediante Fisher. La razón de tasas de incidencia (IRR) se calculó utilizando un análisis de regresión de Poisson y se estimaron las interacciones por año, sexo, edad, diabetes e hipertensión, agregando el término de interacción a los modelos de regresión. Las tasas se calcularon con base en las proyecciones anuales de población del Instituto Nacional de Estadística.

Se reportan valores absolutos y porcentajes para las variables categóricas.

Se calcularon concomitantemente los valores de tiempo desde el inicio de síntomas a la reperfusión y la mortalidad a los 15 días luego del procedimiento.

Las variables continuas se expresan mediante media y la desviación estándar o la mediana y el rango.

Las variables categóricas y cuantitativas se compararon mediante Chi cuadrado y ANOVA, respectivamente.
El estudio fue aprobado por la Junta de revisión del FNR y se obtuvo el consentimiento informado de todos los pacientes antes de realizar cualquier procedimiento de reperfusión, autorizando el uso de sus datos con fines de investigación. El protocolo del estudio se ajusta a las directrices éticas de la Declaración de Helsinki de 1975.

\section{Resultados}

Del 13 de marzo al 30 de abril de 2020 se realizaron en Uruguay un total de 517 ICP (96 electivas y 421 urgentes). Para el mismo período se realizaron 1073 ICP en 2019 (273 electivas y 800 urgentes), 1071 ICP en 2018 (235 electivas y 836 urgentes) y 1052 ICP en 2017 (282 electivas y 770 urgentes).

El número de pacientes tratados por IAMCEST (ICP y FBL) en Uruguay fue menor en 2020 (136 pacientes) en comparación con 2019 (180 pacientes), 2018 (182 pacientes) y 2017 (174 pacientes). De estos, la FBL se realizó como tratamiento único en $5,1 \%, 7,2 \%, 7,7 \%$ y $12,1 \%$ de los casos, respectivamente $(\mathrm{p}>0,05)$.

La tasa de incidencia de IAMCEST durante el período estudiado en 2020 fue de 3,85 (IC del 95\%: 3,23-4,56) por 100.000 personas. Esta tasa fue más baja que la reportada en 2019, 2018 y 2017 (figura 1A).

El análisis de regresión de Poisson mostró que el año 2020 se asoció respecto a 2019 con una IRR de 0,74 (IC del 95\%: 0,59-0,91, p<0,05) para el tratamiento con IAMCEST.

No encontramos diferencias en el tiempo de inicio de los síntomas hasta la reperfusión en comparación con 2019.

La mortalidad a los 15 días de tratamiento por IAMCEST fue similar durante los períodos evaluados en 2017 (8\%), 2018 (6\%), 2019 (11\%) y $2020(8 \%, p=0,422)$.

\section{Discusión}

Este es el primer informe sobre el impacto global a nivel país de la pandemia por SARS-CoV-2 en el tratamiento del IAMCEST. El análisis de la incidencia total del tratamiento con IAMCEST (FBL e ICP) en Uruguay mostró una disminución del 26\% durante los primeros 49 días posteriores a la declaración de emergencia sanitaria en comparación con años anteriores durante el mismo período de tiempo. La mortalidad y el tiempo desde el inicio de los síntomas hasta la reperfusión en estos pacientes fueron similares a los de años anteriores.

Desde el inicio de la pandemia de SARSCoV-2, se han publicado varios informes que po- 


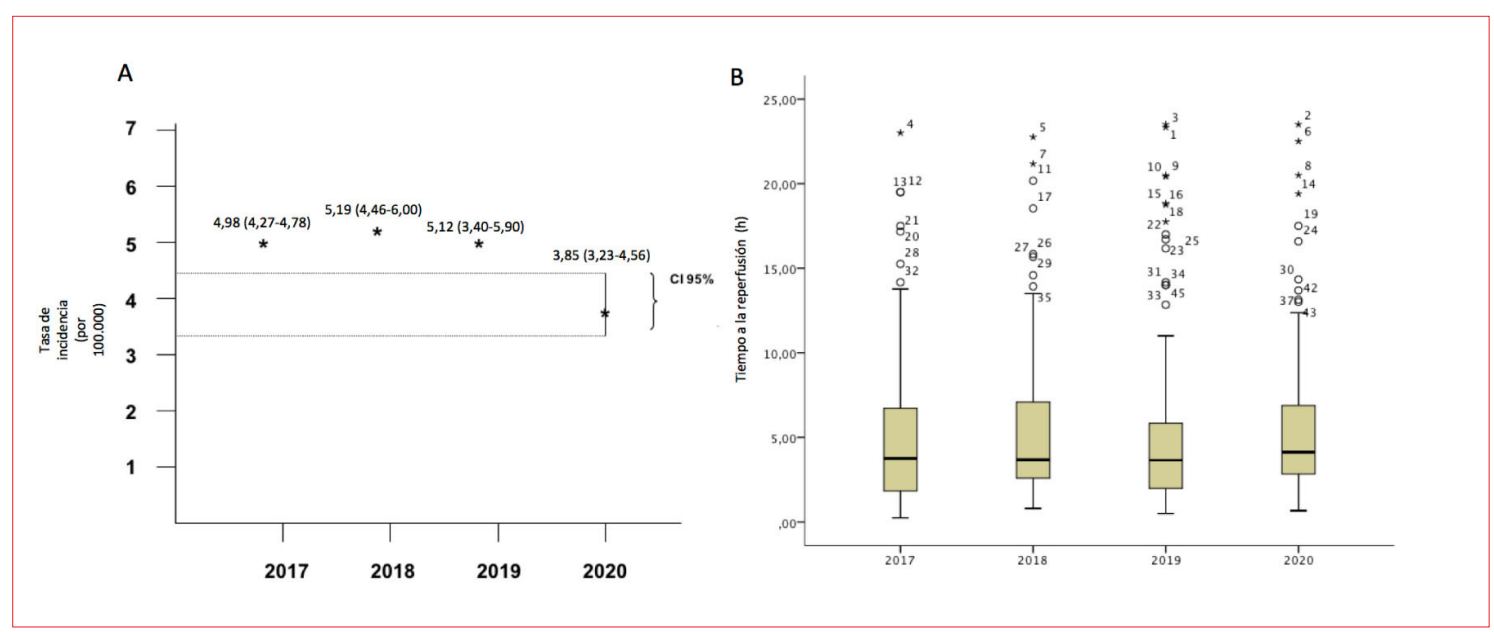

Figura 1. Reperfusión de IAMCEST durante la pandemia de SARS-CoV-2 en Uruguay. A) Tasas de incidencia relativa (IRR) del tratamiento de reperfusión del infarto agudo de miocardio con elevación del ST (IAMCEST) durante el período de tiempo evaluado. El IC del 95\% para 2020 se superpone parcialmente con los IC del 95\% de los años anteriores. B) El tiempo de intervalo desde el inicio de los síntomas hasta la reperfusión para pacientes con IAMCEST durante el período de tiempo evaluado no difirió entre los años $(p=0,4)$. Aunque la mediana del tiempo hasta la reperfusión fue mayor en 2020 (4,10 h; rango 0,67-23,50 h) en comparación con 2019 (3,50 h; rango 0,50-23,50 h), 2018 (3,60 h; rango 0,80-22,75 h) y 2017 ( 3,70 h; rango 0,25-23,00 h), esta diferencia tampoco fue estadísticamente significativa $(p=0,4)$. Los límites superior e inferior de cada caja representan el percentil 75 y 25 , respectivamente, mientras que la línea horizontal representa la mediana. El bigote superior se sitúa por encima del 3er cuartil + entre 1.5 y 3 veces el rango intercuartílico, y el bigote inferior por debajo del 1.er cuartil - 1,5 veces el rango intercuartílico. Los valores que se observan por encima del bigote superior de los diagramas corresponden a los casos codificados que exceden dicho limite, mientras que los asteriscos son valores extremos.

nen alerta sobre la disminución de la incidencia y el tratamiento del IAMCEST. García y colaboradores $^{(1)}$ evaluaron 9 centros de cardiología intervencionista de alto volumen en Estados Unidos y encontraron una reducción del 38\% en la incidencia de IAMCEST. Un gran estudio observacional multicéntrico de Italia mostró que la admisión por IAMCEST durante la pandemia disminuyó un $26,5 \%$, siendo mayor entre las mujeres ${ }^{(5)}$. La tasa de mortalidad informada por los autores casi se triplicó durante la pandemia (del 4,1\% al 13,7\%).

A diferencia de la mayoría de los estudios previos informados sobre este tema, comparamos la incidencia poblacional total y el tratamiento global con IAMCEST (FBL e ICP). Nuestro análisis ha demostrado que la tasa de incidencia del tratamiento con IAMCEST en 2017, 2018 y 2019 fue bastante estable sin un aumento relativo en la terapia con FBL durante el 2020. Considerando que Montevideo abarca la mayoría de los centros de cardiología intervencionista del Uruguay, a diferencia de otros estudios, no encontramos diferencias en la edad, el sexo, el estado hipertenso, la diabetes o la ubicación geográfica entre los pacientes tratados con IAMCEST en 2020 con los años anteriores ${ }^{(5)}$.

A diferencia de Tam y colaboradores ${ }^{(6)}$, no encontramos diferencias en el tiempo desde el inicio de los síntomas hasta la reperfusión durante el período de tiempo evaluado en 2020 en compara- ción con otros años. La mortalidad a 15 días del IAMCEST tratado fue similar a la de años anteriores, lo que nos permite suponer que los pacientes tratados durante la pandemia no tuvieron clínicamente un mayor riesgo. Los datos que reportan De Rosa y colaboradores ${ }^{(6)}$ muestran una mortalidad mucho más alta $(11,9 \%)$ en pacientes ingresados con IAMCEST en Italia. Dos factores pueden ayudar a comprender la diferencia: De Rosa y colaboradores incluyeron a todos los pacientes ingresados en el hospital con IAMCEST (no solo a los tratados con ICP o FBL) y, lo más importante, la sobresaturación del sistema de salud en Italia (algo que no ocurrió en el momento analizado en nuestro país) probablemente haya provocado retrasos en la atención de estos pacientes con la consiguiente mayor mortalidad.

Las pautas chinas para el manejo de STEMI durante la pandemia han recomendado el uso de FBL por encima de $\operatorname{ICP}^{(7)}$. Nuestros datos muestran que la FBL no cambió significativamente con años anteriores y, por lo tanto, no explica la disminución en el tratamiento del IAMCEST por ICP.

Una disminución en las admisiones por IAMCEST se puede explicar de dos formas. Una verdadera disminución en la incidencia de IAMCEST durante la pandemia o las personas pueden haber tenido un IAMCEST y no acceder a los servicios médicos de emergencia. En el último caso, esperaríamos mayores paros cardíacos extrahospitalarios 
(PCEH) y mortalidad cardiovascular durante el período evaluado. Algunos estudios han resaltado una incidencia de PCEH más alta durante la pan$\operatorname{demia}^{(8)}$ y señalaron que los pacientes con SARSCoV-2 representaron una parte importante del aumento. Los datos de nuestro país han demostrado que el número de PCEH no aumentó significativamente durante el período evaluado y los datos del Ministerio de Salud Pública reportaron una disminución significativa del 9\% en la mortalidad global durante el primer semestre del 2020 en Uruguay $^{(9,10)}$. Hasta el momento de redactar el presente manuscrito, no ha habido ningún informe sobre el aumento de la incidencia de la mortalidad cardiovascular durante la pandemia en ningún país en comparación con años anteriores. Hasta entonces, no podemos excluir que la disminución en el número de tratamientos para IAMCEST no se deba a una disminución real en su ocurrencia. La modificación del estilo de vida debido a la cuarentena, la disminución del estrés relacionado con el trabajo y la menor contaminación también podrían ayudar a explicar una disminución en la incidencia de SCA.

\section{Limitaciones}

Nuestro informe no contiene datos sobre mortalidad cardiovascular en Uruguay que puedan ayudar a evaluar la hipótesis de IAMCEST no tratado. Además, no pudimos recolectar datos sobre el número de pacientes ingresados por IAMCEST en cada uno de los principales hospitales de Uruguay debido a las políticas institucionales.

\section{Conclusión}

Las medidas sanitarias de emergencia se asociaron con una disminución en la incidencia de reperfusión de IAMCEST sin afectar el tiempo de reperfusión y la mortalidad. La contribución original de este estudio es que debido a nuestra estructura de salud somos capaces de informar las tasas de incidencia nacionales en el tratamiento de IAMCEST, ya sea por ICP o FBL. Todos los informes anteriores han incluido pacientes en diferentes regiones o estados o conglomerados hospitalarios y han restringido sus datos a ICP. Por lo tanto, no pueden excluir un aumento de FBL como causa probable de disminución de ICP en el tratamiento de IAMCEST. Finalmente, dado que la tasa de incidencia y letalidad por SARS-CoV-2 había sido extremadamente baja hasta la fecha considerada en este estudio, los datos presentados reflejan principalmente el impacto epidemiológico de las medidas sanitarias.
Víctor Dayan, ORCID 0000-0002-5470-0585.

Alejandro Cuesta, ORCID 0000-0003-1315-5581.

Abayubá Perna, ORCID 0000-0001-7062-1310.

Álvaro Niggemeyer, ORCID 0000-0003-0911-6029.

Natalia Piñeiro, ORCID 0000-0002-5238-4494.

Rosana Gambogi, ORCID 0000-0003-0781-7745.

Graciela Fernández, FALTA

Este artículo fue aceptado para su publicación por

Editor jefe anterior, Dr. Gerardo Soca

\section{Bibliografía}

1. Garcia S, Albaghdadi MS, Meraj PM, Schmidt C, Garberich R, Jaffer FA, et al. Reduction in ST-segment elevation cardiac catheterization laboratory activations in the United States during COVID-19 pandemic. J Am Coll Cardiol. 2020; 75 (22):2871-2872. doi: 10.1016/ j.jacc.2020.04.011.

2. De Filippo O, D’Ascenzo F, Angelini F, Bocchino PP, Conrotto F, Saglietto A, et al. Reduced rate of hospital admissions for ACS during Covid-19 outbreak in northern Italy. N Engl J Med. $2020 \mathrm{Jul}$ 2;383(1):88-9. doi:10.1056/NEJMc2009166.

3. Solomon MD, McNulty EJ, Rana JS, Leong TK, Lee C, Sung SH, et al. The Covid-19 pandemic and the incidence of acute myocardial infarction. N Engl J Med. 2020;383(7):691-93. doi:10.1056/NEJMc2015630.

4. Piccolo R, Bruzzese D, Mauro C, Aloia A, Baldi C, Boccalatte M, et al. Population trends in rates of percutaneous coronary revascularization for acute coronary syndromes associated with the COVID-19 outbreak. Circulation. 2020;141(24):2035-2037. doi: 10.1161/ CIRCULATIONAHA.120.047457. Fe de errata en: Circulation. 2020 Jul 14;142(2):e32. doi: 10.1161/ CIR.0000000000000888.

5. De Rosa S, Spaccarotella C, Basso C, Calabrò MP, Curcio A, Filardi PP, et al. Reduction of hospitalizations for myocardial infarction in Italy in COVID-19 era. Eur Heart J. 2020: ehaa409. doi:10.1093/eurheartj/eha a409.

6. Tam CF, Cheung KS, Lam S, Wong A, Yung A, Sze M, et al. Impact of coronavirus disease 2019 (COVID- 19) outbreak on ST-segment-elevation myocardial infarction care in Hong Kong, China. Circ Cardiovasc Qual Outcomes 2020;13(4):e006631. doi: 10.1161/ CIRCOUTCOMES.120.006631.

7. Han Y, Zeng H, Jiang H, Yang Y, Yuan Z, Cheng X, et al. CSC Expert consensus on principles of clinical management of patients with severe emergent cardiovascular diseases during the COVID-19 epidemic. Circulation 2020;141(20):e810-6. doi: 10.1161/CIRCULATIONAHA.120.047011.

8. Marijon E, Karam N, Jost D, Perrot D, Frattini B, Derkenne C, et al. Out-of-hospital cardiac arrest during the COVID-19 pandemic in Paris, France: a population-based, observational study. Lancet Public Health 2020;5(8) e437-43. S2468-2667(20)30117-1.doi: 10.1016/ S24682667(20)30117-1. 
9. Dayan V, Perna A, Piñeiro N, Niggemeyer A. Frecuencia de consultas cardiológicas y paros cardíacos extrahospitalarios durante la pandemia COVID-19. Rev Urug Cardiol. 2020 [consulta: 9 Oct 2021];35(2): [aprox.5p.]. Disponible en: https://ruc. suc.org.uy/ RUC/article/view/140
10. Leon I, Misa A, Gianneo O. Vigilancia de la mortalidad por todas las causas Enero a Julio 2015-2020. Uruguay: Dirección General de Salud. Ministerio de Salud Publica; 2020 [consulta: 9 Oct 2021]. Disponible en: https://www.gub.uy/ministerio-salud-publica/comunicacion/noticias/vigilancia-mortalidad-todas-causas

Contribución de autores

Víctor Dayan: recolección de datos, redacción de borrador, redacción de versión final, correcciones y respuesta a árbitros, análisis estadístico.

Álvaro Niggemeyer: recolección de datos, redacción de borrador, redacción de versión final

Alejandro Cuesta: recolección de datos, redacción de borrador, redacción de versión final

Rosana Gambogi: recolección de datos, redacción de borrador, redacción de versión final

Natalia Pinheiro: recolección de datos, redacción de borrador, redacción de versión final, análisis estadístico.

Abayubá Perna: recolección de datos, redacción de borrador, redacción de versión fina, análisis estadístico. 